\title{
Investimento e satisfação em curso de curta duração: modelo de avaliação
}

\author{
Investment and satisfaction in short-duration \\ course: model of evaluation
}

Newton Kara-Jose', Regina Kazumi Noma ${ }^{2}$, Regina de Souza Carvalho ${ }^{3}$,Miguel Zago Chignalia ${ }^{4}$, Newton Kara Junior ${ }^{5}$

\section{Resumo}

Objetivo: Apresentar um modelo de avaliação da satisfação dos participantes de um curso de curta duração, considerando-se o custo financeiro e o conhecimento adquirido. Métodos: Através da aplicação de questionários, foi realizado um estudo transversal em amostra prontamente acessível, formada por oftalmologistas $(n=29)$ que presenciaram o "II Curso de Transferência de Habilidades em Adaptação de Lentes de Contato", no Hospital Sírio-Libanês (SP). Resultados: A amostra foi composta por 29 indivíduos, $58,6 \%$ do sexo feminino e $37,9 \%$ do sexo masculino (uma pessoa não respondeu à questão). Dos participantes, $41,4 \%$ gastaram menos de 800 reais (U\$400), $24,1 \%$ investiram de 800 a 1000 reais e $34,5 \%$, de 1200 a 3000 reais. A aquisição de conhecimento foi considerada muito satisfatória em 10 (76,92\%) dos itens questionados; em $3(23,08 \%)$, foi avaliada como mediana e, em nenhum caso, foi considerada pequena ou nula. $\mathrm{O}$ curso seria recomendado a outros colegas por $95 \%$ dos participantes.Conclusões: $\mathrm{O}$ modelo apresentado permitiu avaliar a expectativa e o ganho de conhecimento em cada item do programa, a eficiência dos módulos de ensino do curso, os custos para os alunos e a satisfação dos participantes.

Descritores: Educação continuada/economia; Educação médica continuada/economia; Aprendizagem; Satisfação pessoal; Ensino; Cursos; Questionários

\footnotetext{
${ }^{1}$ Professor Titular do Departamento de Oftalmologia do Hospital das Clínicas da Faculdade de Medicina da Universidade de São Paulo e Ciências Médicas da UNICAMP - Campinas (SP), Brasil.

Chefe do Setor de Lentes de Contato do Hospital das Clínicas da Faculdade de Medicina da Universidade de São Paulo - USP - São Paulo (SP), Brasil.

Pedagoga, Pós-graduanda pelo Departamento de Oftalmologia do Hospital das Clínicas da Faculdade de Medicina da Universidade de São Paulo - USP - São Paulo (SP), Brasil.

${ }^{4}$ Residente em Oftalmologia pela Faculdade de Ciências Médicas da Santa Casa de São Paulo - FCMSCSP - São Paulo (SP), Brasil.

${ }^{5}$ Chefe do Setor de Catarata do Hospital das Clínicas da Faculdade de Medicina da Universidade de São Paulo - USP - São Paulo (SP), Brasil.

Trabalho realizado no Hospital das Clínicas da Faculdade de Medicina da Universidade de São Paulo - USP - São Paulo (SP), Brasil. 


\section{INTRODUÇÃO}

$\mathbf{O}$ médico, frente à constante evolução da Medicina e de seu exercício, sempre necessitou da atualização de seus conhecimentos. Recentemente, essa necessidade de reciclagem passou a ser também obrigatória para a revalidação do título de especialista. ${ }^{1}$

De acordo com Dickson $(1996)^{2}$, somente a aprendizagem capacita as pessoas a manterem uma vantagem competitiva a longo prazo, por meio do processamento contínuo das informações. No início da aprendizagem, o conhecimento é novo e tem que ser obtido desde o básico, outras vezes o conhecimento já existe e necessita ser reciclado.

A aprendizagem é um processo de aquisição de conhecimento, que ocorre como resultado da prática e da experiência crítica e que produz uma mudança relativamente permanente no comportamento. ${ }^{3}$

Existe uma relação entre aprendizagem, conhecimento e desempenho, que possibilita satisfazer as necessidades do paciente por meio da oferta de novos serviços e prevenção de complicações. ${ }^{4}$ Esta condição leva a melhores resultados, tais como retenção de clientes, crescimento, lucratividade e sucesso profissional.

Após a formação profissional, o médico faz sua atualização principalmente freqüentando cursos de curta duração.No Brasil, ocorre uma extraordinária oferta desses eventos de extensão, porém contingências de tempo e recursos obrigam o médico a selecionar os que vai participar. ${ }^{6}$

Para uma escolha eficaz, é importante que vários aspectos sejam considerados antes da decisão por um determinado curso, entre eles destacam-se: o programa, a duração, o número de participantes, a interatividade, a credibilidade do corpo docente, a opinião de colegas que já tenham atendido o curso e as despesas diretas e indiretas.

A literatura médica nacional é escassa de modelos de orientação que facilitem a escolha de cursos que venham ao encontro das necessidades do especialista, fornecendo os conhecimentos desejados e que compensem os esforços e recursos aplicados.

O presente trabalho tem por objetivo apresentar um modelo de avaliação da satisfação dos participantes de curso de curta duração, considerando-se o custo financeiro e o conhecimento adquirido.

\section{Métodos}

Foi realizado um estudo transversal entre oftalmologistas presentes no "II Curso de Transferência de Habilidades em Adaptação de Lentes de Contato", dias
10 e 11 de março de 2007, no Hospital Sírio Libanês, em São Paulo.

Este curso, realizado num sábado e domingo, teve duração aproximada de 16 horas, com aulas teóricas, discussões e situações práticas da adaptação de lentes de contato.

Distribuíram-se dois questionários a amostra prontamente acessível, formada por participantes do curso. Os questionários foram preenchidos e devolvidos de maneira voluntária, não havendo necessidade de identificação.

O primeiro questionário foi oferecido no início do curso, para obter características dos participantes (idade, sexo, procedência, escolaridade), gastos para participação no evento, além de quantificar em "muito, mediano, pouco e nada", as expectativas do aluno em relação aos conhecimentos a serem adquiridos.

O segundo questionário foi oferecido ao final do curso para avaliar quanto o aluno aprendeu em relação à expectativa inicial, e se ele indicaria o curso a outros colegas.

O parâmetro para classificação da resposta referente à aquisição de conhecimento com o curso foi estabelecido de maneira aleatória. Assim, os resultados abaixo de $50 \%$ da aquisição de conhecimento foram classificados como pouco satisfatórios; de 50 a $60 \%$, medianos e acima de $60 \%$,muito satisfatórios.

O projeto de pesquisa foi aprovado pela Comissão de Ética do Hospital das Clínicas da Faculdade de Medicina da Universidade de São Paulo no dia 01/08/2007.

\section{Resultados}

A amostra da primeira avaliação foi constituída por 29 oftalmologistas, $17(58,6 \%)$ do sexo feminino e $11(37,9 \%)$ do masculino. Um participante $(3,4 \%)$ não respondeu a essa questão.

A idade variou de 24 a 56 anos, média de 32 anos (desvio padrão 9,25).

Dez $(34,5 \%)$ participantes eram de São Paulo, sendo $4(13,8 \%)$ da capital e $6(20,7 \%)$ do interior do Estado. Seis (20,7\%) vieram de outros estados do sudeste, $7(24,0 \%)$ da região sul e $6(20,7 \%)$ eram procedentes do nordeste.

A maior parte da amostra (55,0\%) era constituída por residentes ou estagiários de Oftalmologia, enquanto $45 \%$ eram representados por profissionais já em prática.

Dos participantes, $58,6 \%$ gastaram mais de 800,00 reais (U\$ 400,00) para participar do curso $(24,1 \%$ in- 
Tabela 1

\begin{abstract}
Expectativa versus conhecimento adquirido no curso "Transferência de Habilidades em Adaptação de Lentes de Contato" (São Paulo, março/2007)
\end{abstract}

\begin{tabular}{|c|c|c|c|c|}
\hline \multirow[t]{2}{*}{$\begin{array}{l}\text { Assunto/ } \\
\text { Grau de Conhecimento }\end{array}$} & \multicolumn{2}{|c|}{$\begin{array}{c}\text { Expectativa } \\
(\mathbf{n}=\mathbf{2 9})^{*}\end{array}$} & \multicolumn{2}{|c|}{$\begin{array}{r}\text { Aquisição } \\
(\mathbf{n}=\mathbf{2 0})^{*}\end{array}$} \\
\hline & f & $\%$ & f & $\%$ \\
\hline \multicolumn{5}{|l|}{ Anamnese } \\
\hline Muito & 21 & 72,4 & 17 & 85 \\
\hline Mediano & 6 & 20,7 & 3 & 15 \\
\hline Pouco & 2 & 6,9 & 0 & 0 \\
\hline Nada & 0 & 0 & 0 & 0 \\
\hline Total & 29 & 100 & 20 & 100 \\
\hline
\end{tabular}

Seleção de pacientes

Muito

Mediano

Pouco

Nada

Total

$\begin{array}{cccc}19 & 65,5 & 14 & 70 \\ 8 & 27,6 & 5 & 25 \\ 2 & 6,9 & 1 & 5 \\ 0 & 0 & 0 & 0 \\ 29 & 100 & 20 & 100\end{array}$

Adaptação de Lentes de Contato

$\begin{array}{lcccc}\text { Muito } & 24 & 82,8 & 16 & 80 \\ \text { Mediano } & 4 & 13,8 & 4 & 20 \\ \text { Pouco } & 1 & 3,4 & 0 & 0 \\ \text { Nada } & 0 & 0 & 0 & 0 \\ \text { Total } & 29 & 100 & 20 & 100\end{array}$

Nomenclatura de Lentes de Contato

Muito

Mediano

$23 \quad 79,3$

Pouco

Nada

Total

$\begin{array}{cc}23 & 79,3 \\ 6 & 20,7 \\ 0 & 0 \\ 0 & 0\end{array}$

14
5
1
0
20

$$
\begin{gathered}
70 \\
25 \\
5 \\
0
\end{gathered}
$$

Tipos de Lentes de Contato

Muito

Mediano

Pouco

Nada

Total

$\begin{array}{cccc}23 & 79,3 & 12 & 60 \\ 4 & 13,8 & 4 & 20 \\ 2 & 6,9 & 4 & 20 \\ 0 & 0 & 0 & 0 \\ 29 & 100 & 20 & 100\end{array}$

*A diferença entre o $\mathrm{n}$ da expectativa e o da aquisição ocorreu porque alguns dos participantes deixaram o curso antecipadamente, devido a horários de vôo para retorno às cidades de origem.

vestiram de 800,00 a 1.000,00 reais e $34,5 \%$, de $1.200,00$ a 3.000,00 reais).

A expectativa pela aquisição de conhecimento e a concretização dessa expectativa variou de acordo com cada item abordado nos questionários e estão relacionadas nas tabelas de 1 a 3. A aquisição de conhecimento foi considerada muito satisfatória em 10 quesitos (Tabelas 1 e 2) e mediana em 3 dos assuntos contemplados (tabela 3). Nenhum quesito teve avaliação de "pouco" ou "nada".
Tabela 2

Expectativa versus conhecimento adquirido no curso "Transferência de Habilidades em Adaptação de Lentes de Contato" (São Paulo, março/2007).

\begin{tabular}{lcccc}
\hline $\begin{array}{l}\text { Assunto/ } \\
\text { Grau de Conhecimento }\end{array}$ & \multicolumn{2}{c}{$\begin{array}{c}\text { Expectativa } \\
(\mathbf{n}=\mathbf{2 9})\end{array}$} & \multicolumn{2}{c}{$\begin{array}{c}\text { Aquisição } \\
(\mathbf{n}=\mathbf{2 0})\end{array}$} \\
& $\mathbf{f}$ & $\mathbf{\%}$ & $\mathbf{f}$ & $\%$ \\
\hline Testes com Lentes de Contato & & & \\
Muito & 25 & 86,2 & 16 & 80 \\
Mediano & 3 & 10,4 & 3 & 15 \\
Pouco & 1 & 3,4 & 1 & 5 \\
Nada & 0 & 0 & 0 & 0 \\
Total & 29 & 100 & 20 & 100 \\
& & & & \\
Dúvidas e Queixas dos Pacientes & & & \\
Muito & 22 & 75,9 & 15 & 75 \\
Mediano & 7 & 24,1 & 4 & 20 \\
Pouco & 0 & 0 & 1 & 5 \\
Nada & 0 & 0 & 0 & 0 \\
Total & 29 & 100 & 20 & 100
\end{tabular}

Educação do Usuário de Lentes de Contato

$\begin{array}{lcccc}\text { Muito } & 21 & 72,4 & 18 & 90 \\ \text { Mediano } & 7 & 24,1 & 1 & 5 \\ \text { Pouco } & 1 & 3,4 & 1 & 5 \\ \text { Nada } & 0 & 0 & 0 & 0 \\ \text { Total } & 29 & 100 & 20 & 100\end{array}$

Como Organizar um Setor de Lentes de Contato $\begin{array}{llll}\text { Muito } & 17 & 58,6 & 12\end{array}$

$\begin{array}{llll}\text { Mediano } & 8 & 27,6 & 7\end{array}$

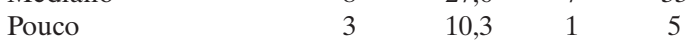

$\begin{array}{lllll}\text { Nada } & 1 & 3,4 & 0 & 0\end{array}$

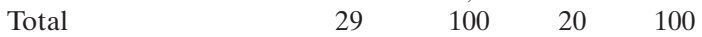

Aspectos Legais na Adaptação de Lentes de Contato $\begin{array}{lllll}\text { Muito } & 19 & 65,5 & 13 & 65\end{array}$

$\begin{array}{lllll}\text { Mediano } & 9 & 31 & 4 & 20\end{array}$

$\begin{array}{lllll}\text { Pouco } & 1 & 3,4 & 1 & 10\end{array}$

Nada $\quad 0 \quad c c c c c$

$\begin{array}{lllll}\text { Total } & 29 & 100 & 20 & 100\end{array}$

*A diferença entre o $\mathrm{n}$ da expectativa e o da aquisição ocorreu porque alguns dos participantes deixaram o curso antecipadamente, devido a horários de vôo para retorno às cidades de origem.

Noventa e cinco por cento dos participantes recomendariam o curso a outros colegas.

\section{Dıscussão}

Embora não se possa duvidar da intenção dos organizadores de cursos de alcançar a melhor qualidade possível, não é usual, em Medicina, a aplicação de métodos de auditoria que ajudem a avaliar os resulta- 
Tabela 3

Expectativa versus conhecimento adquirido no curso "Transferência de Habilidades em Adaptação de Lentes de Contato" (São Paulo, março/2007).

\begin{tabular}{|c|c|c|c|c|}
\hline \multirow[t]{2}{*}{$\begin{array}{l}\text { Assunto/ } \\
\text { Grau de Conhecimento }\end{array}$} & \multicolumn{2}{|c|}{$\begin{array}{c}\text { Expectativa } \\
(\mathbf{n}=29)^{*}\end{array}$} & \multicolumn{2}{|c|}{$\begin{array}{r}\text { Aquisição } \\
(\mathbf{n}=\mathbf{2 0}) *\end{array}$} \\
\hline & $\mathbf{f}$ & $\%$ & $\mathbf{f}$ & $\%$ \\
\hline \multicolumn{5}{|c|}{ Seleção da Lente de Contato } \\
\hline Muito & 23 & 79,3 & 11 & 55 \\
\hline Mediano & 5 & 17,2 & 9 & 45 \\
\hline Pouco & 1 & 3,4 & 0 & 0 \\
\hline Nada & 0 & 0 & 0 & 0 \\
\hline Total & 29 & 100 & 20 & 100 \\
\hline
\end{tabular}

Complicações por Uso de Lentes de Contato

$\begin{array}{lcccc}\text { Muito } & 21 & 72,4 & 11 & 55 \\ \text { Mediano } & 8 & 27,6 & 5 & 25 \\ \text { Pouco } & 0 & 0 & 2 & 10 \\ \text { Nada } & 0 & 0 & 0 & 0 \\ \text { Não Respondeu } & 0 & 0 & 2 & 10 \\ \text { Total } & 29 & 100 & 20 & 100\end{array}$

Como Conquistar e Manter o Paciente no Consultório $\begin{array}{lllll}\text { Muito } & 21 & 72,4 & 10 & 50\end{array}$

$\begin{array}{lllll}\text { Mediano } & 7 & 24,1 & 9 & 45\end{array}$

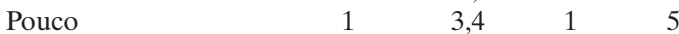

\begin{tabular}{|c|c|}
\hline Nada & 0 \\
\hline
\end{tabular}

$\begin{array}{lllll}\text { Total } & 29 & 100 & 20 & 100\end{array}$

*A diferença entre o $\mathrm{n}$ da expectativa e o da aquisição ocorreu porque alguns dos participantes deixaram o curso antecipadamente, devido a horários de vôo para retorno às cidades de origem.

dos obtidos, considerando a percepção dos alunos.

Por outro lado, não existe método multifatorial para ser aplicado na avaliação da qualidade do ensino que considere a expectativa e os resultados obtidos em cada módulo, associados à avaliação dos gastos (investimento) e da satisfação manifestada pela disposição de indicar o curso para outros colegas. O que geralmente ocorre é a coleta de impressão dos participantes sobre cada aula após os eventos.

O presente trabalho propõe um modelo de avaliação e, para testar sua eficiência, foi escolhido um curso sobre educação em lentes de contato. Apesar do sucesso evidenciado pela procura crescente por cursos de lentes de contato realizados no Hospital das Clínicas e no Hospital Sírio-Libanês, nunca houve avaliação multifatorial da satisfação dos clientes. Assim, as modificações visando o aprimoramento do aprendizado baseiam-se apenas em observações pessoais e nas poucas manifestações de alunos. ${ }^{6}$

Esses cursos, por seu caráter teórico-prático, ofe- recem um número limitado de vagas, e, dos trinta inscritos, apenas um não pôde comparecer por impedimentos de última hora.

Ressalta-se que a participação em um curso envolve tempo, deslocamento, gastos diretos e indiretos, e por vezes, o esforço não é recompensado. Cada vez mais o profissional necessita escolher, entre as diferentes ofertas de reuniões científicas, aquelas que correspondam às suas expectativas (custo-benefício). Deve basear-se em: possível aquisição de conhecimentos, programa, carga horária, credibilidade dos docentes, parecer de colegas que já freqüentaram o curso anteriormente e custo financeiro direto e indireto.

No curso analisado, os alunos ainda despenderam um fim de semana e a maioria viajou longa distância para atender ao curso, gastando "só em espécie" entre 800 a 3000 reais (aproximadamente 400 a 1500 dólares). Note-se que o custo teria sido maior se o evento fosse realizado durante dias úteis da semana, quando haveria o adicional do lucro cessante.

A decisão dos participantes de arcar com o investimento financeiro provavelmente associa-se à grande expectativa de aprendizado manifestado na pesquisa (tabelas 1 a 3). Expectativa tem papel fundamental na expressão da satisfação e depende da experiência anterior acumulada, pois a satisfação está diretamente relacionada com ganho cognitivo. O grau de satisfação depende da percepção dos benefícios obtidos e de quanto os resultados se aproximaram das expectativas. ${ }^{7} \mathrm{~A}$ amostra estudada foi de residentes e médicos já em prática, e, como tal, possuidores de experiência anterior para saber o que procurar em um curso de instrução. Logo, a amostra tem excelente capacidade crítica para avaliar o conhecimento desejado e o alcançado.

Os questionários mostraram que, em dez dos treze quesitos estudados, a percepção dos alunos foi avaliada como muito satisfatória quanto à aquisição de conhecimento (Tabelas 1 e 2). Alguns quesitos (Tabela 3) tiveram avaliação mediana. A identificação dos pontos que necessitam ser aperfeiçoados em cursos futuros auxilia o organizador de cursos na busca pela excelência e pelo cumprimento das expectativas de todos os envolvidos: clientes, docentes e organizadores.

Noventa e cinco por cento dos participantes desta pesquisa revelaram a disposição de recomendar esse curso para outros colegas. Essa atitude evidenciou o alto grau de satisfação alcançada.

A obtenção de clientela satisfeita é fundamental para que os cursos tradicionais com aluno presente mantenham sua continuidade, pois os progressos contínuos 
na área de comunicação têm possibilitado cada vez mais o emprego de telecomunicação e outras formas de ensino a distância. ${ }^{8}$ Essas modalidades de educação apresentam uma série de vantagens e desvantagens. Entre suas vantagens estão: menor ou nenhum deslocamento, facilidades de horário, menor custo direto e indireto. E, entre as desvantagens, está a menor interação dos participantes dos cursos com os outros alunos e com os docentes. Os cursos presenciais sofrerão impacto negativo quanto à participação de congressistas e deverão se aprimorar muito para enfrentar essa concorrência; apenas os cursos mais eficientes terão chances de continuar existindo com sucesso.

O modelo aplicado permitiu comparar a expectativa com a obtenção de conhecimentos nos diferentes módulos do curso; permitiu, ainda, avaliar a percepção dos alunos quanto ao custo-benefício da participação no evento e se ele merece ser recomendado para outros colegas.

\section{Conclusões}

O modelo apresentado permitiu: avaliar a expectativa e o ganho alcançado nos diferentes itens do programa; evidenciar os módulos de ensino percebidos como mais ou menos efetivos; avaliar os custos despendidos pelos alunos e a satisfação dos participantes do curso. A aplicação deste modelo mostrou-se eficiente para a orientação de reestruturação de cursos de extensão.

\section{AbStract}

Objective: To present a model of assessment of the satisfaction level of the attendants in a short-duration course, considering the financial costs and the acquired knowledge.Methods: By means of the presentation of questionnaires, a transversal study on a promptly accessible sample of ophthalmologists $(n=29)$ who attended the "II Course on Transfer of Skills on Adaptation to Contact Lenses", at Sirio-Libanês Hospital, was carried out. Results: The sample was formed by 29 individuals, being 58,6\% men and 37,9\% women (one person did not answered to the question). $41.4 \%$ of the attendants have spent less than 800 reais (US\$ 400,00); 24.1\% have invested from 800 up to 1000 reais; and 34,5\%, from 1200 up to 3000 reais. The acquisition of knowledge was considered "very satisfactory" in relation to 10 (76.92\%) of the researched items; "average" in relation to other 3 (23.08\%); and no item was considered insufficient. The course would be recommended to colleagues by $95 \%$ of the attendants. Conclusions: The model allowed the assessment of expectations and the acquisition of knowledge in each item of the program, the efficiency of each panel of the course, the costs for the students and thesatisfaction level of the attendants.

Keywords: Education, continuing/economics ; Education, medical, continuing/economics; Learning; Personal satisfaction, Teaching, Courses; Questionnaires

\section{REFERÊNCIAS}

1. Conselho Federal de Medicina. Resolução CFM N ${ }^{\circ}$ 1.772/ 2005. Institui o Certificado de Atualização Profissional para os portadores dos títulos de especialista e certificados de áreas de atuação e cria a Comissão Nacional de Acreditação para elaborar normas e regulamentos para este fim, além de coordenar a emissão desses certificados [Internet]. Diário Oficial da República, Federativa do Brasil: Brasília (DF); 2005 Ago 8, Seção I , p. 141- 2 [citado 2007 Dez 12]. Disponível em: http://www.cna-cap.org.br/resolucao.php4.

2. Dickson PR. The static and dynamic mechanics of competition: a comment on Hunt and Morgan's comparative advantage theory. J Mark. 1996; 60(4):102-6.

3 Caravantes GR, Bretas Pereira M. Aprendizagem organizacional versus estratégia de mudança organizacional planejada: um confronto crítico. Porto Alegre: FAPERGS; 1985.

4. Day GS. The capabilities of market-driven organizations. J Mark. 1994; 58(4):37-52.

5. Meireles M, Scarpi MJ. Estudo do impacto de um curso MBA Gestão em Saúde na modernidade organizacional de clínicas oftalmológicas. Arq Bras Oftalmol. 2005; 68(6): 757-67.

6. Carvalho RS, Temporini ER, Kara-José N, Noma R, Bechara SJ, Marback R. Percepções de oftalmologistas referentes ao modelo de ensino aplicado a lentes de contato. Arq Bras Oftalmol. 2006; 69(4):615-9.

7. Sitzia J, Wood N. Patient satisfaction: a review of issues and concepts. Soc Sci Med. 1997; 45(12):1829-43. Comment in: Birth. 2008; 35(1): 73-6.

8. Kirigia JM, Sambo LG, Phiri M, Matsembula G, Awases M. Cost-effectiveness analysis of establishing a distanceeducation programme for health personnel in Swaziland. Afr J Health Sci. 2002; 9(1-2):3-15.

\section{Endereço para correspondência}

Av. Dr. Enéas de Carvalho Aguiar, 255. Cerqueira

César. São Paulo.

e-mail: miguelzc@bol.com.br 